\title{
Development of polyethylene films coated with gelatin and mango peel extract and the effect on the quality of margarine
}

\begin{abstract}
Polyethylene (PE) films coated with an active layer of gelatin and mango peel extract (MPE) were developed for margarine product. MPE as an antioxidant source was incorporated into a fish gelatin-based film-forming solution (FFS). The FFS was coated onto PE films with different thicknesses $(10,20,40$, and $60 \mu \mathrm{m})$ to form polyethylene/gelatin bilayer films (PE/G). The physical and antioxidant properties of the PE/G films were determined. Thicker coatings produced coloured films, which improved light barrier properties and increased DPPH radical scavenging values. Scanning electron microscopy (SEM) analysis showed the bilayer films were compatible with one another and produced compacted film integrity. Also, the PE/G60 film improved the oxidation stability of margarine up to 28 days of storage period at $4{ }^{\circ} \mathrm{C}$. The results suggest that bilayer films have potentials to be used as active packaging materials for delaying lipid oxidation.
\end{abstract}

Keyword: Active packaging; Bilayer film; Shelf life; Mango peel; Antioxidant 